\title{
Evidencia de Uso del Perro (Canis lupus familiaris) en un Contexto Ritual (Tiwanaku, Bolivia)
}

\author{
Evidence of Use of the Dog (Canis Lupus Familiaris) in a Ritual Context (Tiwanaku, \\ Bolivia)
}

\author{
Velia Verónica Mendoza España ${ }^{\mathrm{i}}$
}

\section{RESUMEN}

En la actualidad son escasos los trabajos acerca del manejo ritual de fauna en la arqueología practicada en Bolivia.Análisis arqueofaunísticos, que involucran perros, revelaron en su mayoría un uso económico y algunos indicios, poco claros, de un manejo ritual. En el Proyecto Arqueológico Jach'a Marka 2007 se encontró un ejemplar de Canis lupus familiaris en el Centro Cívico Ceremonial de Tiwanaku, específicamente en el montículo de Mollo Kontu que presenta características de un manejo ritual aproximadamente en el siglo $X$ de nuestra era. El análisis arqueofaunístico revela la presencia de un golpe y cortes en lugares estratégicos del esqueleto que junto a la condición etaria, la posición del individuo y el contexto arqueológico, proporcionan datos fehacientes de un manejo ritual, simbólico y afectivo del perro dentro de la sociedad tiwanacota.

Palabras Claves: Perro, Tiwanaku, Mollo Kontu, Ritual.

\begin{abstract}
Today there is a scant research into the ritual use of animals in Bolivian archaeology.Archaeofaunal analyses of canine remains have primarily revealed the dog's economic role, along with some, albeit unclear, indications of its ritual role. During the 2007 field season of Proyecto Arqueológico Jach'a Marka, excavators encountered the remains of an individual Canis lupus familiaris within the Mollo Kontu mound, inside Tiwanaku's civic center, which were indicative of its having been involved in ritual practice during the $10^{\text {th }}$ century AD. Archaeofaunal analysis of the skeleton has revealed the presence of blunt force trauma. Furthermore, cuts in strategic locations on the skeleton, the dog's age group, the position of its body, and the archaeological context provide strong evidence of the dog's ritual, symbolic, and affective roles within Tiwanaku society.
\end{abstract}

Key Words: Dog, Tiwanaku, Mollo Kontu, Ritual

i Laboratorio de Zooarqueología, Universidad Mayor de San Andrés, Bolivia. Correo-e: velia_arqueologia@yahoo.com 


\section{INTRODUCCIÓN}

El uso ritual y simbólico del perro en Tiwanaku fue poco estudiado, su manejo como ofrenda y todas las connotaciones simbólicas que conlleva son complejas y han sido encaradas, en este trabajo, desde el análisis arqueofaunístico al apoyo con escasa iconografía cerámica y datos arqueológicos. A través de esta investigación se determinó que el manejo ritual del perro tiene que ver con la edad, sexo, asociación con otros animales y relación con humanos de corta edad. Además de la asociación con material cerámico, la posición y orientación del espécimen, así como del tipo de sacrificio y mutilación de un elemento anatómico para simbolizar la compañía al otro mundo o la protección en el paso a la otra vida y la solicitud de lluvias en época de sequía. Este complejo manejo se llevó a cabo en una estructura aterrazada conocida como el montículo de Mollo Kontu que cumplió una función principalmente ceremonial al inicio de una época decadente del Estado Tiwanaku.

\section{SITIO ARQUEOLÓGICO MONTÍCULO DE MOLLO KONTU}

El área de Mollo Kontu se encuentra 250 metros al sur del Centro Cívico Ceremonial de Tiwanaku, Provincia Ingavi, departamento de La Paz, Bolivia (Figura I). El año 2007 se excavaron tres sectores: A) Área mortuoria ubicada al extremo sureste del sitio, D) Área residencial que se encuentra al sureste del montículo y M) Área de una concentración de entierros asociados al muro del edificio ubicado al extremo norte del montículo (Couture et al. 2008). Este último sector es de nuestro interés por hallarse el esqueleto de perro (Canis lupus familiaris) en la unidad MI, evento M-I4 (Locus II23).

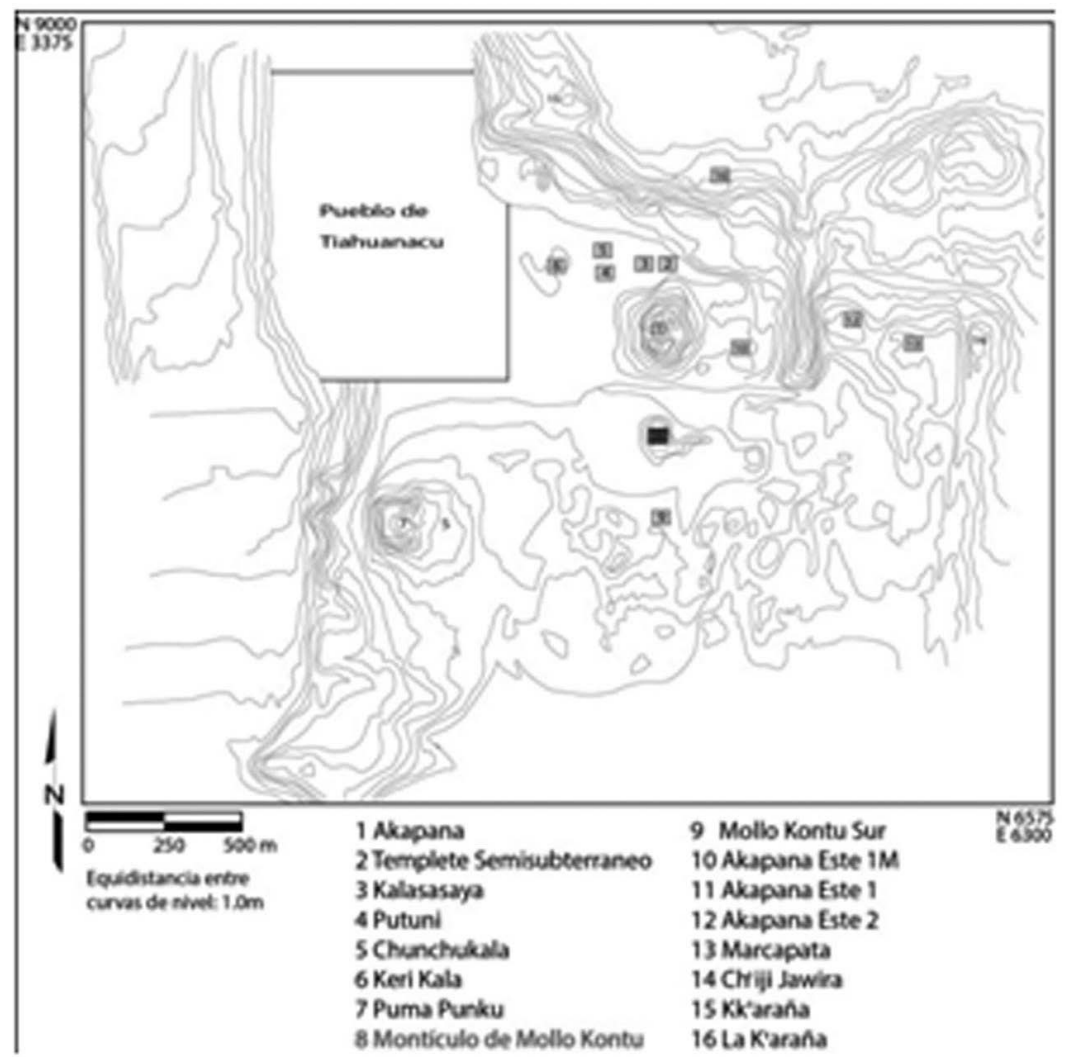

Figura 1: Ubicación del montículo de Mollo Kontu (Modificado de Couture et al. 2008).

Figure 1: Localization of Mollo Kontu mound (Modified of Couture et al. 2008). 


\section{MÉTODO}

La identificación taxonómica del cánido se llevó a cabo mediante su comparación con las colecciones de referencia del Laboratorio de Zooarqueología de la UMSA y la tesis de licenciatura de Mendoza (2004). La estimación de la edad se realizó a partir de la erupción, cambio dental y osificación de los huesos (Valadez 1996, Blanco et al. 2009). La determinación de sexo estuvo limitada por el factor etario, ya que este espécimen se encontraba todavía en desarrollo. A pesar de esto, se realizaron comparaciones con colecciones de referencia de neonatos a juveniles con criterios establecidos por Blanco et al. (2009) que plantean una aproximación al sexo. También se aplicó osteometría, a pesar de la corta edad del espécimen se consideró tomar las medidas de los elementos anatómicos mejor conservados según la metodología establecida por Meadow (2000) y Von den Driesch (1976). Algunas de estas medidas sirvieron para calcular la talla aproximada del espécimen según los criterios propuestos por Valadez (2004).

El análisis tafonómico se realizó mediante la observación macroscópica de cada elemento esqueletal con la ayuda de una lupa, en base a los trabajos de Valadez (2004) y Gutiérrez M. (Com. Pers. 2009). Para la interpretación se consideraron: la escasa iconografía cerámica de perros, datos etnohistóricos y datos de hallazgos arqueológicos similares.

\section{RESULTADOS}

El cánido de Mollo Kontu se encontró en buen estado de conservación, así se pudo determinar que corresponde a un perro con pelo (Canis lupus familiaris) (Figura 2). Su esqueleto está completo en un $85 \%$, su edad aproximada es de cinco a cinco y medio meses, considerándose un ejemplar juvenil. Posiblemente se trate de una hembra, pero por la corta edad no se puede afirmar el sexo. El ejemplar en vida fue de talla pequeña, siendo su alzada de 290 $\mathrm{mm}$. Corresponde al tipo morfológico Jinchuliwi que se caracteriza por presentar cráneo braquicéfalo a mesocéfalo, orejas caídas, cuerpo semi robusto y simétrico, tamaño que va de pequeño a grande, establecido por Mendoza (2004).

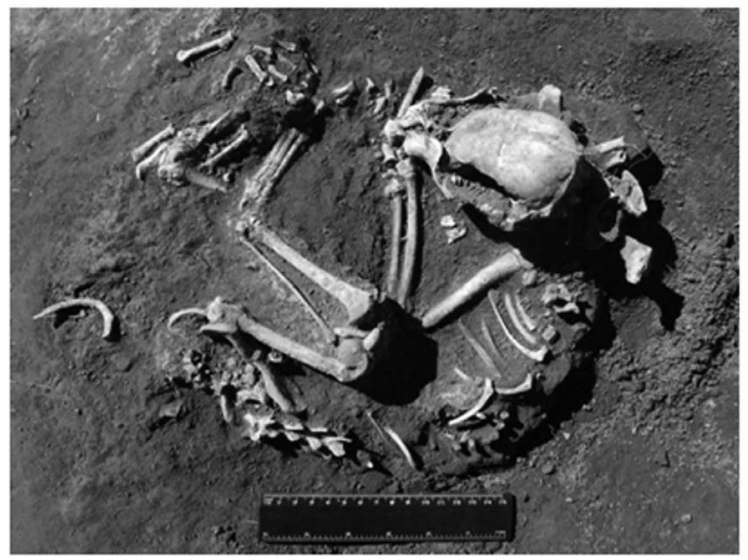

Figura 2: Posición in situ del perro de Mollo Kontu.

Figure 2: Position in situ of the Mollo Kontu dog.

Vale la pena destacar que este ejemplar carece de los primeros premolares (derecho superior y derecho e izquierdo inferior) característica de un carácter congénito común que comparten los perros americanos (Crockford 2005).

Los restos óseos presentan una pequeña huella de corte contundente en la parte distal del cúbito izquierdo y otra más grande en la parte baja del frontal izquierdo (Figura 3).

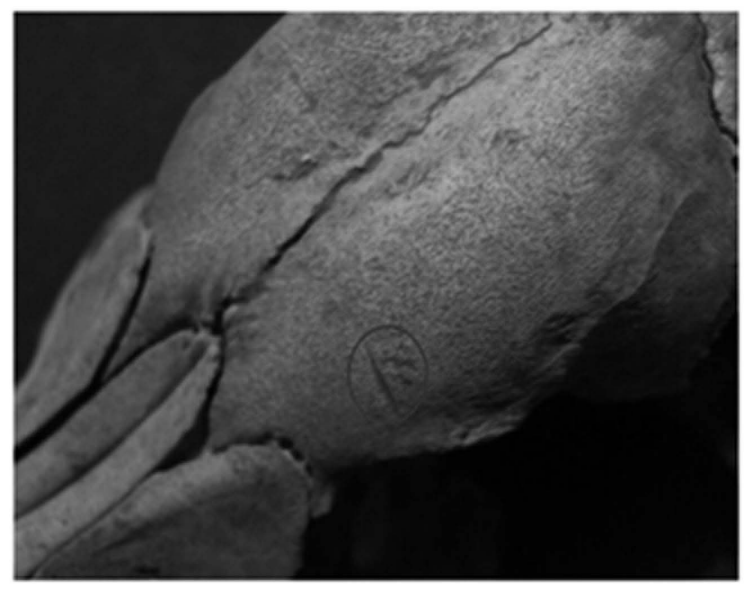

Figura 3: Marca de golpe con un objeto con filo, posible hacha, en el hueso frontal izquierdo.

Figure 3. Presence of blunt force trauma whit the axe in the frontal bone left side.

El espécimen se encontraba asociado a un esqueleto incompleto articulado de anuro y una vasija de cerámica fragmentada con motivo zoomorfo (cabeza de cóndor) que corresponde a un keru.Todo esto relacionado a esqueletos de niños (posibles 
ofrendas) que corresponden al evento "Resto de óseo humano de neonato" caracterizado por no presentar ningún tipo de entierro formal, esqueletos incompletos en mal estado de conservación, siendo parte de los nueve esqueletos humanos incompletos encontrados en esta área (Couture et al. 2008). La posición in situ del perro era de descanso (Figura 2) actualmente conocida como enroscada.

Según el Informe de excavación, el espécimen fue colocado en el perímetro exterior norte de la terraza inferior del montículo, después del abandono del mismo. Se lo encontró orientado de sureste a noroeste. Cronológicamente corresponde al período Tiwanaku IV a V.

\section{CONCLUSIONES}

Entre los años 900 a 1100 d.C. en la parte norte del montículo de Mollo Kontu -Tiwanaku, después del abandono de la estructura, se ofrendó un perro muy joven, posiblemente hembra de talla pequeña con características físicas muy similares al perro de orejas caídas de los dibujos de la crónica de Guaman Poma de Ayala (1988). Fue sacrificado por un golpe contundente en la sien, y posiblemente le cortaron la pata delantera izquierda para llevarla a otro lugar y darle otro uso como la pata de cánido que se halló sobre un esqueleto humano en el templo de Puma Punku (Com. Pers. Yeager J. 2003). Este manejo simbólico apoya la creencia del perro como acompañante a la otra vida: la posición de descanso, la orientación del cuerpo, la asociación con un anuro, según la cosmovisión andina está relacionada a los dioses de las lluvias y la iconografía cerámica de cóndor simboliza al poder. Además de su relación con ofrendas de niños, corrobora su importancia simbólica con la solicitud de lluvia en un momento de sequía en el altiplano (aprox. 900 a II 00 d.C.) (Pierre 199I). La práctica ritual de hacer aullar perros en ceremonias para pedir que llueva se dio en época Inka, según las crónicas del siglo $X V I$ y XVII.

Algunas características del rito de Mollo Kontu las comparte la ofrenda de un perro depositado a la salida de un importante canal de desagüe, en desuso, de la pirámide de Akapana, cuando esta quedó abandonada, en la misma época de sequia (Kolata 1993) compartiendo también la evidencia de ausencia de patas, ya que según Manzanilla (I992:37) “... este curiosamente se encontraba sin hocico ni patas".

La relación de los perros jóvenes con niños humanos se corrobora en la iconografía cerámica Tiwanaku que muestra a un personaje humano cargando un perrito muy pequeño, probablemente neonato, al cual le da de comer o lo acaricia. Otros antecedentes de restos óseos de perros neonatos asociados con restos humanos de jóvenes se encuentran presentes en cistas tiwanaku, existiendo una continuidad de esta práctica hasta el período pos tiwanaku (Mendoza 2004).

Agradecimientos: Un agradecimiento especial a las Directoras del Proyecto Jach'a Marka Dras. Nicole Couture, Deborah Blom y Maria Bruno por permitirme usar sus datos, a los arqueólogos Ruth Fontenla por facilitarme el acceso a los informes y Jonah Augustine por la traducción del resumen. A María del Carmen Mendoza por su colaboración en la redacción y el resto de las traducciones. A Erwin Cortez† por brindarme siempre su apoyo.

\section{BIBLIOGRAFÍA}

Blanco A., B. Rodríguez y R. Valadez. 2009. Estudio de los cánidos arqueológicos del México prehispánico, Instituto Nacional de Antropología e Historia, Universidad Nacional Autónoma de México, Instituto de Investigaciones Antropológicas, México D.F.

Couture N., D. Blom y M. Bruno. 2008. Proyecto Arqueológico Jach'a Marka Informe de Investigaciones realizadas el 2007, La Paz, Bolivia.

Crockford S. 2005. El perro lanudo de Norteamérica. Memorias del 30th World Congress México, México city.

Guaman Poma de Ayala, F. 1988 [1615] El Primer Nueva Corónica y Buen Gobierno. Editores John V. Murra y Rolena Adorno (Traducción Jorge L. Urioste), Tomo I, II, II. 2da edición. Editorial Siglo XXI. México.

Kolata A. 1993. The Tiwanaku: Portrait of an Andean Civilization, Backell Cambridge MA \&Oxford UK.

Manzanilla L. 1992. Akapana: Una pirámide en el centro del mundo. UNAM. IIA. México.

Meadow, R. 2000. "The contributions of Barbara Lawrence to the study of dogs, with a comprehensive listing of her measurement definitions". Procedings of the Ist ICAZ Symposium on the History of the Domestic Dog, 35-50,Victoria, B.C., Canada.

Mendoza, V. 2004. El perro en las sociedades andinas del pasado: Un aporte arqueozoológico (Del Formativo al Inkario. Altiplano norte de Bolivia).Tesis para optar al grado de Licenciatura en Arqueología, Carrera de Arqueología, Universidad Mayor de San Andrés, La Paz, Bolivia. 
Pierre, Y. 1991. "Los paisajes lacustres antiguos según el análisis palinológico”, El Lago Titicaca. Síntesis del conocimiento limnológico actual, Claude Dejoux y André Iltis editores, ORSTOM, Hisbol, La Paz - Bolivia.

Valadez R. 1996. "Anatomía dental del perro pelón mexicano. Estudio odontológico comparativo del Xoloitzcuintle". AMMVEPE 7 (5): 179-183.

Valadez R., L. Gamboa, N. Vélez, B. Rodríguez, M. Gómez, R. García y G. Pérez. 2004. "Perros y prácticas rituales en una antigua aldea de la cuenca de México". AMMVEPE 15(5): I58-I7I.

Valliéres C., E. Arratia y V. Mendoza. 2009.“Estudios Zooarqueológicos en Mollo Kontu - Tiwanaku Proyecto Jach'a Marka". Congreso de Arqueología Boliviana, Universidad Mayor de San Andrés, La Paz, en prensa.

Von den Driesch, A. 1976, A guide to the measurement of animal bones from archaeological sites, Peabody Museum Bulletin I Peabody of Archaeology and Ethnology, Harvard, University. 